\title{
VARIANTS OF KREISEL'S CONJECTURE ON A NEW NOTION OF PROVABILITY
}

\author{
PAULO GUILHERME SANTOS AND REINHARD KAHLE
}

\begin{abstract}
Kreisel's conjecture is the statement: if, for all $n \in \mathbb{N}$, PA $\vdash_{k \text { steps }} \varphi(\bar{n})$, then PA $\vdash \forall x . \varphi(x)$. For a theory of arithmetic $T$, given a recursive function $h, T \vdash<_{h} \varphi$ holds if there is a proof of $\varphi$ in $T$ whose code is at most $h(\# \varphi)$. This notion depends on the underlying coding. $P_{T}^{h}(x)$ is a predicate for $\vdash_{<h}$ in $T$. It is shown that there exist a sentence $\varphi$ and a total recursive function $h$ such that $T \vdash<h \operatorname{Pr}_{T}\left(\left\ulcorner\operatorname{Pr}_{T}(\ulcorner\varphi\urcorner) \rightarrow \varphi\right\urcorner\right)$, but $T \vdash \measuredangle h \varphi$, where $\operatorname{Pr}_{T}$ stands for the standard provability predicate in $T$. This statement is related to a conjecture by Montagna. Also variants and weakenings of Kreisel's conjecture are studied. By the use of reflexion principles, one can obtain a theory $T_{\Gamma}^{h}$ that extends $T$ such that a version of Kreisel's conjecture holds: given a recursive function $h$ and $\varphi(x)$ a $\Gamma$-formula (where $\Gamma$ is an arbitrarily fixed class of formulas) such that, for all $n \in \mathbb{N}, T \vdash_{\leq h} \varphi(\bar{n})$, then $T_{\Gamma}^{h} \vdash \forall x . \varphi(x)$. Derivability conditions are studied for a theory to satisfy the following implication: if $T \vdash \forall x . P_{T}^{h}(\ulcorner\varphi(\dot{x})\urcorner)$, then $T \vdash \forall x . \varphi(x)$. This corresponds to an arithmetization of Kreisel's conjecture. It is shown that, for certain theories, there exists a function $h$ such that $\vdash_{k \text { steps }} \subseteq \vdash_{\leq h}$.
\end{abstract}

\$1. Preliminaries. Let $T$ be a fixed theory of arithmetic which is a consistent primitive-recursive extension of Robinson's Q.

Following [14], we say that a partial function $f: \mathbb{N}^{k} \rightarrow \mathbb{N}$ is strongly representable in $T$ if there is a formula $\varphi\left(x_{0}, \ldots, x_{k-1}, y\right)$ such that ${ }^{1}$

(i) For all $m_{0}, \ldots, m_{k-1}, n \in \mathbb{N}, f\left(m_{0}, \ldots, m_{k-1}\right) \simeq n \Longleftrightarrow T \vdash \varphi\left(\overline{m_{0}}, \ldots\right.$, $\left.\overline{m_{k-1}}, \bar{n}\right)$

(ii) $T \vdash \forall x_{0} \cdots \forall x_{k-1} \cdot \exists ! y \cdot \varphi\left(x_{0}, \ldots, x_{k-1}, y\right)$.

If a function $f$ is strongly representable by a $\Sigma_{1}$-formula $\varphi(x, y)$ in $T$, then $f$ is provably recursive in $T$ (see [13] for a definition of this last concept). Every theory of arithmetic which is a consistent primitive-recursive extension of $\mathrm{Q}$ can strongly represent every (partial) recursive function (not necessarily by a $\Sigma_{1}$-formula; see [14]).

For a standard Gödelization $\ulcorner$.$\urcorner of the underlying language, we use$ Feferman's dot notation [16, p. 837]: Let $\operatorname{sub}(x, y)$ be a function-symbol such that, for every term $t, T \vdash \operatorname{sub}(\ulcorner\varphi\urcorner,\ulcorner t\urcorner)=\ulcorner\varphi(t)\urcorner$, and let num $(x)$

Received March 6, 2020.

2020 Mathematics Subject Classification. 03F30, 03F03.

Key words and phrases. Kreisel's conjecture, provability predicate, formal arithmetic.

${ }^{1} \bar{n}$ is the standard representation of the number $n$ in the theory $T$.

(C) The Author(s), 2021. Published by Cambridge University Press on behalf of Association for Symbolic Logic. This is an Open Access article, distributed under the terms of the Creative Commons Attribution licence (https://creativecommons.org/licenses/by/4.0/), which permits unrestricted re-use, distribution, and reproduction in any medium, provided the original work is properly cited. 
be a function-symbol which represents the numerals in $T$. With $\mathrm{s}(x, y):=$ $\operatorname{sub}(x, \operatorname{num}(y))$ we denote $\mathrm{s}(\ulcorner\varphi(x)\urcorner, y)$ by $\ulcorner\varphi(\dot{y})\urcorner$.

$P(x)$ is a provability predicate in $T$ if for all formulas $\varphi, T \vdash P(\ulcorner\varphi\urcorner)$ if, and only if, $\varphi$ is a theorem of $T$. Furthermore, $S(x)$ is a notion of provability in $T$ if, for every formula $\varphi, T \vdash S(\ulcorner\varphi\urcorner)$ implies that $\varphi$ is provable in $T$. For a provability predicate $P(x)$, we define $\operatorname{Con}_{P}:=\neg P(\ulcorner\perp\urcorner)$.

We say a formula is $\Sigma_{n}$ when it is equivalent in $T$ to a $\Sigma_{n}$-formula.

For a (partial) recursive function $h$, the notation $T \vdash_{<h} \varphi$ expresses that $h(\# \varphi)$ is defined and $\varphi$ is provable in $T$ with a proof whose code is at most $h(\# \varphi)$. This notion generalises the approach followed in [9, pp. 33-35]. $\vdash_{\leq h}$ depends heavily on the chosen Gödelization: different codings give rise to different notions. For the rest the concrete Gödelization is assumed to be a fixed one.

Given $T$, the theory $K_{T}$ extends $T$ by the following axiom schema:

Axiom K. If $f$ is a total recursive function such that, for all $n \in \mathbb{N}$, $f(n) \neq 0$, and $\mathrm{R}(x, y)$ is a formula that strongly represents $f$ in $T$, then $K_{T} \vdash \forall x . \neg \mathrm{R}(x, 0)$.

This schema can be restricted to a smaller class of functions in such a way that $K_{T}$ is recursively enumerable (for instance by considering the class of all primitive recursive functions [10]).

§2. Introduction. According to [5], Kreisel's conjecture is the statement:

If, for all $n \in \mathbb{N}$, PA $\vdash_{k \text { steps }} \varphi(\bar{n})$, then PA $\vdash \forall x . \varphi(x)$.

Kreisel's conjecture has been studied for different systems, with partial solutions for specific theories of arithmetic other than PA - see, for instance, $[1,11,12]$; for a detailed account on the conjecture we refer to [2].

In this paper we present results similar to Kreisel's conjecture for $\vdash_{\leq h}$, which are not restricted to PA. It is an interesting feature of our approach that it does not depend so heavily on the particular axiomatisation of $T$ that one chooses. In some sense, it can be seen as a uniform approach, since it applies to any consistent, primitive-recursive extension of Q.

For $h$ be a total recursive function, the adapted Kreisel's conjecture for $\vdash_{\leq h}$ is:

For all $n \in \mathbb{N}, T \vdash_{\leq h} \varphi(\bar{n})$, then $T \vdash \forall x . \varphi(x)$.

THEOREM 2.1. The adapted Kreisel's conjecture for $\vdash_{\leq h}$ is false.

Proof. Let Proof $(x, y)$ be a standard proof predicate of $T$ that expresses that $x$ is a proof of $y$ (see [16, p. 838] for details). Let $h$ be the function defined by:

$h(m):= \begin{cases}\mu k[k \text { is the code of a proof of the formula coded by } m \text { in } T], \\ \text { if } m=\neg \operatorname{Proof}(\bar{n},\ulcorner\perp\urcorner), \text { for some } n, \\ \text { otherwise, }\end{cases}$ 
where $\mu$ denotes the minimisation function (see [16, p. 833] for further details on minimisation). It is clear that $h$ is a (total) recursive function. By construction, for all $n \in \mathbb{N}, T \vdash_{<h} \neg \operatorname{Proof}(\bar{n},\ulcorner\perp\urcorner)$. If the adapted Kreisel's conjecture for $\vdash_{\leq h}$ was true, it would follow $T \vdash \forall x . \neg \operatorname{Proof}(x,\ulcorner\perp\urcorner)$, contradicting the second incompleteness theorem (see [16, p. 828] for further information).

It is not known whether the theorem still holds if one restricts oneself to primitive-recursive functions.

The result is in accordance with [6], where several reasons are given to believe that Kreisel's conjecture is, in fact, false.

Even though the adapted Kreisel's conjecture for $\vdash_{\leq h}$ is false, it is worth studying variants and weakenings of it. For example, one could ask for an extension $T^{h}$ of $T$ such that Kreisel's conjecture holds adapted to $T^{h}$ : given a total recursive function $h$, if, for all $n \in \mathbb{N}, T \vdash_{\leq h} \varphi(\bar{n})$, then $T^{h} \vdash \forall x . \varphi(x)$.

One immediate solution would be to add the true sentence $\forall x . \varphi(x)$ as an axiom to $T$. We will, however, construct a theory $T^{h}$, avoiding the trivial $a$ priori addition of $\forall x . \varphi(x)$ as an axiom. The approach is of interest, because it allows to establish relations between different concepts.

We will also study versions of the conjecture for theories that satisfy certain derivability conditions. We exhibit conditions for a theory to satisfy the following implication:

$$
\text { If } T \vdash \forall x . P_{T}^{h}(\ulcorner\varphi(\dot{x})\urcorner) \text {, then } T \vdash \forall x . \varphi(x) \text {. }
$$

This corresponds to an arithmetization of Kreisel's conjecture.

Finally, we prove, for certain theories, the existence of a total recursive function $h$ such that $\vdash_{k \text { steps }} \subseteq \vdash_{\leq h}$.

§3. On the notion of provability $\vdash_{\leq h}$. In this section, we study the notion $\vdash_{\leq h}$ and some of its properties. We start with a result that guarantees that $\vdash \leq h$ is representable in $T$.

THEOREM 3.1. Given a total recursive function $h$, there is a notion of provability $P_{T}^{h}(x)$ that represents $\vdash_{\leq h}$ in $T$ such that if, for all $n \in \mathbb{N}$, $T \vdash_{\leq h} \alpha(\bar{n})$, then $K_{T} \vdash \forall x . P_{T}^{h}(\ulcorner\alpha(\dot{x})\urcorner)$.

PROOF. Let $h$ be an arbitrary, but fixed total recursive function. We define $f_{h}$ by:

$f_{h}(n):= \begin{cases}\mu m \leq h(n)[m \text { is the code of a proof of the formula coded by } n], & \begin{array}{l}\text { if } n \text { is a code of a formula and there is such an } m, \\ \text { otherwise. }\end{array}\end{cases}$

$f_{h}$ is a total recursive function, thus $f_{h}$ can be strongly representable by a formula $\mathrm{R}_{h}(x, y)$ in $T$. Given $n, m \in \mathbb{N}$, it is clear that $m \leq h(n)$ is the smallest code of a proof of the formula coded by $n$ if, and only if, $T \vdash$ $\mathrm{R}_{h}(\bar{n}, \bar{m}) \wedge \bar{m} \neq 0$. Thus, we can define the provability predicate $P_{T}^{h}(x):=$ $\exists y \neq 0 . \mathbf{R}_{h}(x, y)$. 
Assume that, for all $n \in \mathbb{N}, T \vdash_{\leq h} \alpha(\bar{n})$. Let $g_{h}$ be the function defined by: $g_{h}(n):=f_{h}(\# \alpha(\bar{n})) . g_{h}$ is a total recursive function. Furthermore, $g_{h}$ is strongly representable by the formula $\mathrm{S}_{h}(x, y):=\mathrm{R}_{h}(\ulcorner\alpha(\dot{x})\urcorner, y)$ since:

(i) If $g_{h}(n)=m$, then $f_{h}(\# \alpha(\bar{n}))=m$, and thus $T \vdash \mathrm{R}_{h}(\ulcorner\alpha(\bar{n})\urcorner, \bar{m})$, i.e., $T \vdash \mathrm{S}_{h}(\bar{n}, \bar{m})$;

(ii) As $T \vdash \forall x . \exists ! y . \mathbf{R}_{h}(x, y)$ it follows that $T \vdash \forall x . \exists ! y \cdot \mathbf{R}_{h}(\ulcorner\alpha(\dot{x})\urcorner, y)$, i.e., $T \vdash \forall x . \exists ! y . \mathrm{S}_{h}(x, y)$.

By hypothesis, for all $n \in \mathbb{N}$, there is $m \leq h(\# \alpha(\bar{n}))$ such that $m$ is the code of a proof of $\alpha(\bar{n})$ in $T$. Hence, for all $n \in \mathbb{N}, g_{h}(n) \neq 0$. As $\mathrm{S}_{h}(x, y)$ strongly represents $g_{h}$, we have by hypothesis that $K_{T} \vdash \forall x . \neg S_{h}(x, 0)$. From $T \vdash \forall x . \exists ! y . \mathrm{S}_{h}(x, y)$ follows that $T \vdash \forall x . \exists y . \mathrm{S}_{h}(x, y)$. Together with $K_{T} \vdash$ $\forall x . \neg S_{h}(x, 0)$, it follows that $K_{T} \vdash \forall x . \exists y \neq 0 . S_{h}(x, y)$, i.e., $K_{T} \vdash \forall x . \exists y \neq$ $0 . \mathrm{R}_{h}(\ulcorner\alpha(\dot{x})\urcorner, y)$. So, $K_{T} \vdash \forall x . P_{T}^{h}(\ulcorner\alpha(\dot{x})\urcorner)$.

We show that, for all formulas $\varphi, T \vdash_{\leq h} \varphi \Longleftrightarrow T \vdash P_{T}^{h}(\ulcorner\varphi\urcorner)$. Suppose that $T \vdash_{\leq h} \varphi$. For $m:=f_{h}(\# \varphi)$, we have that $m \neq 0$. Thus, $T \vdash \mathrm{R}_{h}(\ulcorner\varphi\urcorner, \bar{m}) \wedge \bar{m} \neq 0$, and so $T \vdash \exists y \neq 0 . \mathrm{R}_{h}(\ulcorner\varphi\urcorner, y)$. Hence, $T \vdash$ $P_{T}^{h}(\ulcorner\varphi\urcorner)$. Now suppose that $T \vdash P_{T}^{h}(\ulcorner\varphi\urcorner)$. Let $m:=f_{h}(\# \varphi)$. If $m \neq 0$, then $T \vdash_{\leq h} \varphi$. Suppose, towards a contradiction, that $m=0$. We have that $T \vdash \exists y \neq 0 . \mathbf{R}_{h}(\ulcorner\varphi\urcorner, y)$ and $T \vdash \mathrm{R}_{h}(\ulcorner\varphi\urcorner, 0)$. As $T \vdash \forall x . \exists ! y . \mathrm{R}_{h}(x, y)$ we arrive at a contradiction. So, $m \neq 0$, as desired.

The next result follows immediately from the proof of the last theorem.

Corollary 3.1. Given a total recursive function $h$, and a formula $\varphi$, we have that $T \vdash_{\leq h} \varphi \Longleftrightarrow T \vdash P_{T}^{h}(\ulcorner\varphi\urcorner)$.

The provability predicate $P_{T}^{h}$ is provably decidable, in the following sense:

THEOREM 3.2. Given a total recursive function $h$, for every formula $\varphi$, we have that $T \vdash P_{T}^{h}(\ulcorner\varphi\urcorner)$ or $T \vdash \neg P_{T}^{h}(\ulcorner\varphi\urcorner)$.

Proof. Suppose that $T \nvdash P_{T}^{h}(\ulcorner\varphi\urcorner)$. By the previous result, $T \vdash \leq h \varphi$. This means that $f_{h}(\# \varphi)=0$. As $\mathrm{R}_{h}(x, y)$ strongly represents the function $f_{h}$, it follows that $T \vdash \mathrm{R}_{h}(\ulcorner\varphi\urcorner, 0)$. Since $T \vdash \forall x . \exists ! y . \mathrm{R}_{h}(x, y)$, we can conclude that $T \vdash \forall y .\left(\mathrm{R}_{h}(\ulcorner\varphi\urcorner, y) \rightarrow y=0\right)$, and so, $T \vdash \neg \exists y .\left(y \neq 0 \wedge \mathrm{R}_{h}(\ulcorner\varphi\urcorner, y)\right)$, i.e., $T \vdash \neg P_{T}^{h}(\ulcorner\varphi\urcorner)$.

The next result corresponds to an arithmetization of the previous statement.

THEOREM 3.3. Given a provability predicate $P(x)$ and a total recursive function $h$, we have for every formula $\varphi$ that $T \vdash P\left(\left\ulcorner P_{T}^{h}(\ulcorner\varphi\urcorner)\right\urcorner\right) \vee$ $P\left(\left\ulcorner\neg P_{T}^{h}(\ulcorner\varphi\urcorner)\right\urcorner\right)$.

Proof. From Theorem 3.2, it follows $T \vdash P_{T}^{h}(\ulcorner\varphi\urcorner)$ or $T \vdash \neg P_{T}^{h}(\ulcorner\varphi\urcorner)$. If $T \vdash P_{T}^{h}(\ulcorner\varphi\urcorner)$, then $T \vdash P\left(\left\ulcorner P_{T}^{h}(\ulcorner\varphi\urcorner)\right\urcorner\right)$, and so $T \vdash P\left(\left\ulcorner P_{T}^{h}(\ulcorner\varphi\urcorner)\right\urcorner\right) \vee$ $P\left(\left\ulcorner\neg P_{T}^{h}(\ulcorner\varphi\urcorner)\right\urcorner\right)$. If $T \vdash \neg P_{T}^{h}(\ulcorner\varphi\urcorner)$, then $T \vdash P\left(\left\ulcorner\neg P_{T}^{h}(\ulcorner\varphi\urcorner)\right\urcorner\right)$; hence $T \vdash P\left(\left\ulcorner P_{T}^{h}(\ulcorner\varphi\urcorner)\right\urcorner\right) \vee P\left(\left\ulcorner\neg P_{T}^{h}(\ulcorner\varphi\urcorner)\right\urcorner\right)$. In $\quad$ sum, $\quad T \vdash P\left(\left\ulcorner P_{T}^{h}(\ulcorner\varphi\urcorner)\right\urcorner\right) \vee$ $P\left(\left\ulcorner\neg P_{T}^{h}(\ulcorner\varphi\urcorner)\right\urcorner\right)$. 
We now prove that there is no $h$ such that $\vdash_{\leq h}$ coincides with $\vdash$.

THEOREM 3.4. For every total recursive function $h$, there is a formula $\varphi$ such that $T \vdash \varphi$, but $T \vdash \leq h \varphi$.

Proof. Let $h$ be a fixed total recursive function. Let $\varphi$ be the sentence obtained from the application of the diagonalisation lemma [15, p. 169] to the formula $\neg P_{T}^{h}(x)$. Then,

$$
T \vdash \varphi \leftrightarrow \neg P_{T}^{h}(\ulcorner\varphi\urcorner) .
$$

Suppose, towards a contradiction, that $T \vdash_{\leq h} \varphi$. Thus, $T \vdash \varphi$. By Corollary 3.1 we have that $T \vdash P_{T}^{h}(\ulcorner\varphi\urcorner)$, and so, by $(\mathrm{I}), T \vdash \neg \varphi$, which contradicts $T \vdash \varphi$. Hence, $T \vdash \leq h \varphi$. From Theorem 3.2 it follows that $T \vdash \neg P_{T}^{h}(\ulcorner\varphi\urcorner)$, i.e., $T \vdash \varphi$.

The next fact will play a major role for the discussion of Kreisel's conjecture.

THEOREM 3.5. Given a total recursive function $h$, for every formula $\varphi, T \vdash$ $P_{T}^{h}(\ulcorner\varphi\urcorner) \rightarrow \varphi$.

Proof. Let $\varphi$ be an arbitrary formula. From Theorem 3.2, $T \vdash P_{T}^{h}(\ulcorner\varphi\urcorner)$ or $T \vdash \neg P_{T}^{h}(\ulcorner\varphi\urcorner)$. First, suppose that $T \vdash P_{T}^{h}(\ulcorner\varphi\urcorner)$. Then, by Corollary 3.1, we conclude $T \vdash \leq h \varphi$, from where we get $T \vdash \varphi$. Thus, $T \vdash P_{T}^{h}(\ulcorner\varphi\urcorner) \rightarrow \varphi$. Second, suppose that $T \vdash \neg P_{T}^{h}(\ulcorner\varphi\urcorner)$. Then, by logic, $T \vdash P_{T}^{h}(\ulcorner\varphi\urcorner) \rightarrow \varphi$. In all, $T \vdash P_{T}^{h}(\ulcorner\varphi\urcorner) \rightarrow \varphi$.

THEOREM 3.6. Let $h$ be a primitive-recursive function and $P(x)$ be a provability predicate such that:

C1 For all $\Sigma_{1}$-formulas $\varphi, T \vdash \varphi \rightarrow P(\ulcorner\varphi\urcorner)$;

C2 For all formulas $\varphi$ and $\psi, T \vdash \varphi \rightarrow \psi \Longrightarrow T \vdash P(\ulcorner\varphi\urcorner) \rightarrow P(\ulcorner\psi\urcorner)$.

Then, for every formula $\varphi, T \vdash \neg P\left(\left\ulcorner\neg P_{T}^{h}(\ulcorner\varphi\urcorner)\right\urcorner\right) \rightarrow P_{T}^{h}(\ulcorner\varphi\urcorner)$.

Proof. If $h$ is primitive-recursive, then $\mathrm{R}_{h}(x, y)$ can be picked as being a $\Sigma_{1}$-formula. Clearly, $T \vdash \neg P_{T}^{h}(\ulcorner\varphi\urcorner) \leftrightarrow \mathrm{R}_{h}(\ulcorner\varphi\urcorner, 0)$. From $\mathbf{C 2}$ we get that $T \vdash P\left(\left\ulcorner\neg P_{T}^{h}(\ulcorner\varphi\urcorner)\right\urcorner\right) \leftrightarrow P\left(\left\ulcorner\mathrm{R}_{h}(\ulcorner\varphi\urcorner, 0)\right\urcorner\right)$. From C1, $T \vdash \mathrm{R}_{h}(\ulcorner\varphi\urcorner, 0) \rightarrow$ $P\left(\left\ulcorner\mathrm{R}_{h}(\ulcorner\varphi\urcorner, 0)\right\urcorner\right)$, so $T \vdash \neg P_{T}^{h}(\ulcorner\varphi\urcorner) \rightarrow P\left(\left\ulcorner\neg P_{T}^{h}(\ulcorner\varphi\urcorner)\right\urcorner\right)$, as wanted.

Let $\operatorname{Pr}_{T}(x):=\exists y . \operatorname{Proof}(x, y)$ denote the standard provability predicate in $T\left[16\right.$, p. 826] and $\operatorname{Con}_{T}:=\neg \operatorname{Pr}_{T}(\ulcorner\perp\urcorner)$.

THEOREM 3.7. Given a primitive-recursive function $h$, for every formula $\varphi$, $T+\operatorname{Con}_{T} \vdash \operatorname{Pr}_{T}\left(\left\ulcorner P_{T}^{h}(\ulcorner\varphi\urcorner)\right\urcorner\right) \rightarrow P_{T}^{h}(\ulcorner\varphi\urcorner)$.

Proof. It is clear that $T \vdash \operatorname{Pr}_{T}\left(\left\ulcorner P_{T}^{h}(\ulcorner\varphi\urcorner)\right\urcorner\right) \wedge \operatorname{Pr}_{T}\left(\left\ulcorner\neg P_{T}^{h}(\ulcorner\varphi\urcorner)\right\urcorner\right) \rightarrow$ $\operatorname{Pr}_{T}(\ulcorner\perp\urcorner)$. Thus, $T+\operatorname{Con}_{T} \vdash \operatorname{Pr}_{T}\left(\left\ulcorner P P_{T}^{h}(\ulcorner\varphi\urcorner)\right\urcorner\right) \wedge \operatorname{Pr}_{T}\left(\left\ulcorner\neg P_{T}^{h}(\ulcorner\varphi\urcorner)\right\urcorner\right) \rightarrow \perp$. Hence, $T+\operatorname{Con}_{T} \vdash \neg \operatorname{Pr}_{T}\left(\left\ulcorner P_{T}^{h}(\ulcorner\varphi\urcorner)\right\urcorner\right) \vee \neg \operatorname{Pr}_{T}\left(\left\ulcorner\neg P_{T}^{h}(\ulcorner\varphi\urcorner)\right\urcorner\right)$, i.e., $T+$ $\operatorname{Con}_{T} \vdash \operatorname{Pr}_{T}\left(\left\ulcorner P_{T}^{h}(\ulcorner\varphi\urcorner)\right\urcorner\right) \rightarrow \neg \operatorname{Pr}_{T}\left(\left\ulcorner\neg P_{T}^{h}(\ulcorner\varphi\urcorner)\right\urcorner\right)$. By the previous result we conclude that $T+\operatorname{Con}_{T} \vdash \operatorname{Pr}_{T}\left(\left\ulcorner P_{T}^{h}(\ulcorner\varphi\urcorner)\right\urcorner\right) \rightarrow P_{T}^{h}(\ulcorner\varphi\urcorner)$. 
§4. Montagna's conjecture. Löb's theorem [9, pp. 28-29] expresses that for all formulas $\varphi$, if $T \vdash \operatorname{Pr}_{T}(\ulcorner\varphi\urcorner) \rightarrow \varphi$, then $T \vdash \varphi$. More generally, for all formulas $\varphi$,

$$
T \vdash \operatorname{Pr}_{T}\left(\left\ulcorner\operatorname{Pr}_{T}(\ulcorner\varphi\urcorner) \rightarrow \varphi\right\urcorner\right) \leftrightarrow \operatorname{Pr}_{T}(\ulcorner\varphi\urcorner) .
$$

If one analyses the proof of Löb's theorem, it indicates that one can prove $\operatorname{Pr}_{T}(\ulcorner\varphi\urcorner) \rightarrow \varphi$ only if one has already proved $\varphi$. It indicates, moreover, that $\operatorname{Pr}_{T}\left(\left\ulcorner\operatorname{Pr}_{T}(\ulcorner\varphi\urcorner) \rightarrow \varphi\right\urcorner\right)$ is only provable, if $\varphi$ is proven in the first place. This intuition can be related to a problem proposed by Montagna in [3, $\mathrm{p}$. 9]: “Does PA $\vdash_{k \text { steps }} \operatorname{Pr}_{\mathrm{PA}}(\ulcorner\varphi\urcorner) \rightarrow \varphi$ imply PA $\vdash_{k \text { steps }} \varphi$ ?" Let us consider a variant of this question: "Does $T \vdash_{k \text { steps }} \operatorname{Pr}_{T}\left(\left\ulcorner\operatorname{Pr}_{T}(\ulcorner\varphi\urcorner) \rightarrow \varphi\right\urcorner\right)$ imply $T \vdash_{k \text { steps }} \varphi$ ?" We prove that the adapted question to the provability notion $\vdash_{\leq h}$ is false: " $T \vdash_{\leq h} \operatorname{Pr}_{T}\left(\left\ulcorner\operatorname{Pr}_{T}(\ulcorner\varphi\urcorner) \rightarrow \varphi\right\urcorner\right)$ does not imply $T \vdash_{\leq h} \varphi$."

THEOREM 4.1. For every primitive-recursive function $g(x, y)$ with $g(x, y)>$ $y$, there are a sentence $\varphi$ and a number $n_{0}$ such that $T \vdash_{\leq h} \operatorname{Pr}_{T}\left(\left\ulcorner\operatorname{Pr}_{T}(\ulcorner\varphi\urcorner) \rightarrow\right.\right.$ $\varphi\urcorner)$, but $T \vdash \leq h \varphi$, where $h:=\lambda x . g\left(x, n_{0}\right)$.

PRoof. We follow closely the proof of Theorem 14 from [9, p. 34]. Let $g$ be a function-symbol that represents the primitive-recursive function $g$. By the diagonalization lemma, there is a sentence $\varphi$ such that

$$
\begin{array}{r}
T \vdash \varphi \leftrightarrow \exists y .\left(\operatorname{Proof}\left(\left\ulcorner\operatorname{Pr}_{T}\left(\left\ulcorner\operatorname{Pr}_{T}(\ulcorner\varphi\urcorner) \rightarrow \varphi\right\urcorner\right)\right\urcorner, y\right)\right. \\
\wedge \forall z \leq \mathrm{g}(\ulcorner\varphi\urcorner, y) . \neg \operatorname{Proof}(\ulcorner\varphi\urcorner, z)) .
\end{array}
$$

By construction, $\quad T+\operatorname{Pr}_{T}\left(\left\ulcorner\operatorname{Pr}_{T}\left(\left\ulcorner\operatorname{Pr}_{T}(\ulcorner\varphi\urcorner) \rightarrow \varphi\right\urcorner\right)\right\urcorner\right)+\neg \operatorname{Pr}_{T}(\ulcorner\varphi\urcorner) \vdash \varphi$. As $T$ is $\Sigma_{1}$-complete, $T+\varphi \vdash \operatorname{Pr}_{T}(\ulcorner\varphi\urcorner)$. Thus, we can conclude that $T+$ $\operatorname{Pr}_{T}\left(\left\ulcorner\operatorname{Pr}_{T}\left(\left\ulcorner\operatorname{Pr}_{T}(\ulcorner\varphi\urcorner) \rightarrow \varphi\right\urcorner\right)\right\urcorner\right) \vdash \operatorname{Pr}_{T}(\ulcorner\varphi\urcorner)$. By Löb's theorem, it follows that $\quad T \vdash \operatorname{Pr}_{T}\left(\left\ulcorner\operatorname{Pr}_{T}\left(\left\ulcorner\operatorname{Pr}_{T}(\ulcorner\varphi\urcorner) \rightarrow \varphi\right\urcorner\right)\right\urcorner\right) \leftrightarrow \operatorname{Pr}_{T}\left(\left\ulcorner\operatorname{Pr}_{T}(\ulcorner\varphi\urcorner)\right\urcorner\right)$. Hence, $T+\operatorname{Pr}_{T}\left(\left\ulcorner\operatorname{Pr}_{T}(\ulcorner\varphi\urcorner)\right\urcorner\right) \vdash \operatorname{Pr}_{T}(\ulcorner\varphi\urcorner)$. Again by Löb's theorem, it follows that $T \vdash \operatorname{Pr}_{T}(\ulcorner\varphi\urcorner)$, and consequently $T \vdash \varphi$ and $T \vdash \operatorname{Pr}_{T}\left(\left\ulcorner\operatorname{Pr}_{T}(\ulcorner\varphi\urcorner) \rightarrow \varphi\right\urcorner\right)$. This means that $\varphi$ is true. Let $n_{0}$ satisfy the true existential property of $\varphi$. Then, $n_{0}$ is the code of a proof of $\operatorname{Pr}_{T}\left(\left\ulcorner\operatorname{Pr}_{T}(\ulcorner\varphi\urcorner) \rightarrow \varphi\right\urcorner\right)$. By hypothesis on $g$, it follows that $n_{0}<g\left(\operatorname{PPr}_{T}\left(\left\ulcorner\operatorname{Pr}_{T}(\ulcorner\varphi\urcorner) \rightarrow \varphi\right\urcorner\right), n_{0}\right)=$ $h\left(\# \operatorname{Pr}_{T}\left(\left\ulcorner\operatorname{Pr}_{T}(\ulcorner\varphi\urcorner) \rightarrow \varphi\right\urcorner\right)\right)$, ergo $T \vdash_{\leq h} \operatorname{Pr}_{T}\left(\left\ulcorner\operatorname{Pr}_{T}(\ulcorner\varphi\urcorner) \rightarrow \varphi\right\urcorner\right)$. From the fact that $\varphi$ is true one can conclude that for all $z \leq g\left(\# \varphi, n_{0}\right), z$ is not the code of a proof of $\varphi$. This means that $T \vdash \leq h \varphi$.

If a formula $\varphi$ is provable in $T$, we define

$$
\|\varphi\|_{T}:=\min \{n \mid n \text { is the code of a proof of } \varphi \text { in } T\} .
$$

Moreover, if $\varphi$ and $\psi$ are formulas, we stipulate that $\varphi<_{T} \psi$ if $T \vdash \varphi \wedge$ $\psi$ and $\|\varphi\|_{T}<\|\psi\|_{T}$. The following result confirms that the mentioned intuition that a proof of $\operatorname{Pr}_{T}\left(\left\ulcorner\operatorname{Pr}_{T}(\ulcorner\varphi\urcorner) \rightarrow \varphi\right\urcorner\right)$ should encompass, in a way, a proof of $\varphi$ fails.

THEOREM 4.2. There is a formula $\varphi$ such that

$$
\operatorname{Pr}_{T}\left(\left\ulcorner\operatorname{Pr}_{T}(\ulcorner\varphi\urcorner) \rightarrow \varphi\right\urcorner\right)<_{T} \varphi .
$$


Proof. By the diagonalization lemma, there is a sentence $\varphi$ such that

$$
\begin{gathered}
T \vdash \varphi \leftrightarrow \exists y .\left(\operatorname{Proof}\left(\left\ulcorner\operatorname{Pr}_{T}\left(\left\ulcorner\operatorname{Pr}_{T}(\ulcorner\varphi\urcorner) \rightarrow \varphi\right\urcorner\right)\right\urcorner, y\right)\right. \\
\wedge \forall z \leq y . \neg \operatorname{Proof}(\ulcorner\varphi\urcorner, z)) .
\end{gathered}
$$

Applying the same reasoning as in the previous proof, it follows that $T \vdash$ $\operatorname{Pr}_{T}\left(\left\ulcorner\operatorname{Pr}_{T}(\ulcorner\varphi\urcorner) \rightarrow \varphi\right\urcorner\right) \wedge \varphi$; in particular $\varphi$ is true. Take $n_{0}$ as being the natural number that is guaranteed to exist from the true formula $\varphi$. It is straightforward that $\left\|\operatorname{Pr}_{T}\left(\left\ulcorner\operatorname{Pr}_{T}(\ulcorner\varphi\urcorner) \rightarrow \varphi\right\urcorner\right)\right\|_{T} \leq n_{0}$. As $\varphi$ is true, it follows that for all $z \leq n_{0}, z$ is not the code of a proof of $\varphi$. Hence, $n_{0}<\|\varphi\|_{T}$, and so $\left\|\operatorname{Pr}_{T}\left(\left\ulcorner\operatorname{Pr}_{T}(\ulcorner\varphi\urcorner) \rightarrow \varphi\right\urcorner\right)\right\|_{T}<\|\varphi\|_{T}$. In all, $\operatorname{Pr}_{T}\left(\left\ulcorner\operatorname{Pr}_{T}(\ulcorner\varphi\urcorner) \rightarrow \varphi\right\urcorner\right)<_{T}$ $\varphi$.

§5. Variants of Kreisel's conjecture. In this section we present some partial results related to Kreisel's conjecture, namely variants of the conjecture for provability predicates in the present of different derivability conditions. In this section, the theory $T$ does not need to be r.e.

THEOREM 5.1. Let $h$ be a primitive-recursive function and $T$ be such that there is a provability predicate $P(x)$ satisfying:

C1 If $\varphi(x)$ is a $\Sigma_{n}$-formula, then $T \vdash \varphi(x) \rightarrow P(\ulcorner\varphi(\dot{x})\urcorner)$;

C2 $T \vdash P_{T}^{h}(\ulcorner\varphi(\dot{x})\urcorner) \rightarrow P(\ulcorner\varphi(\dot{x})\urcorner)$;

C3 For all formulas $\varphi(x)$ and $\psi(x), \quad T \vdash P(\ulcorner\varphi(\dot{x}) \rightarrow \psi(\dot{x})\urcorner) \rightarrow$ $(P(\ulcorner\varphi(\dot{x})\urcorner) \rightarrow P(\ulcorner\psi(\dot{x})\urcorner))$.

If $\varphi(x)$ is a $\Pi_{n}$-formula such that $T \vdash \forall x . P_{T}^{h}(\ulcorner\varphi(\dot{x})\urcorner)$, then $T+C_{P} \vdash$ $\forall x . \varphi(x)$.

Proof. As $\varphi(x)$ is $\Pi_{n}$, by $\mathbf{C 1}$, we have $T \vdash \neg \varphi(x) \rightarrow P(\ulcorner\neg \varphi(\dot{x})\urcorner)$. Thus, $T \vdash \exists x . \neg \varphi(x) \rightarrow \exists x . P(\ulcorner\neg \varphi(\dot{x})\urcorner)$. By $\mathbf{C 2}, T \vdash P_{T}^{h}(\ulcorner\varphi(\dot{x})\urcorner) \rightarrow P(\ulcorner\varphi(\dot{x})\urcorner)$. Hence, $T \vdash \forall x . P_{T}^{h}(\ulcorner\varphi(\dot{x})\urcorner) \rightarrow \forall x . P(\ulcorner\varphi(\dot{x})\urcorner)$. So, $T+\forall x . P_{T}^{h}(\ulcorner\varphi(\dot{x})\urcorner) \wedge$ $\exists x . \neg \varphi(x) \vdash \exists x . P(\ulcorner\neg \varphi(\dot{x})\urcorner) \wedge \forall x . P(\ulcorner\varphi(\dot{x})\urcorner)$. By C3, $T+\forall x . P_{T}^{h}(\ulcorner\varphi(\dot{x})\urcorner) \wedge$ $\exists x . \neg \varphi(x) \vdash P(\ulcorner\perp\urcorner)$, i.e., $T+\operatorname{Con}_{P} \vdash \forall x . P_{T}^{h}(\ulcorner\varphi(\dot{x})\urcorner) \rightarrow \forall x . \varphi(x)$.

The condition $T \vdash \forall x . P_{T}^{h}(\ulcorner\varphi(\dot{x})\urcorner)$ corresponds to an arithmetization of the antecedent of a version of Kreisel's conjecture. Thus, the result is weaker than Kreisel's conjecture. If $T \vdash \mathrm{Con}_{P}$, then the previous result can be proved inside $T$. The next result is a particular case of the previous theorem.

COROLLARY 5.1. Let $h$ be a primitive-recursive function. If $\varphi(x)$ is a $\Pi_{1}$ formula such that $T \vdash \forall x . P_{P A}^{h}(\ulcorner\varphi(\dot{x})\urcorner)$, then $P A+\operatorname{Con}_{P A} \vdash \forall x . \varphi(x)$.

PROOF. The corollary follows immediately from the fact that $\operatorname{Pr}_{\mathrm{PA}}$ satisfies $\mathbf{C 1}$ and $\mathbf{C 2}$ of the previous theorem [7].

THEOREM 5.2. Let $h$ be a primitive-recursive function and $T$ be such that there is a provability predicate $P(x)$ satisfying:

C1 For all formulas $\varphi(x), T \vdash P(\ulcorner\neg \varphi(\dot{x})\urcorner) \rightarrow P\left(\left\ulcorner\neg P_{T}^{h}(\ulcorner\varphi(\dot{x})\urcorner)\right\urcorner\right)$; 
C2 If $\varphi(x)$ is a $\Sigma_{n}$-formula, then $T \vdash \varphi(x) \rightarrow P(\ulcorner\varphi(\dot{x})\urcorner)$;

C3 For all formulas $\varphi(x)$ and $\psi(x), \quad T \vdash P(\ulcorner\varphi(\dot{x}) \rightarrow \psi(\dot{x})\urcorner) \rightarrow$ $(P(\ulcorner\varphi(\dot{x})\urcorner) \rightarrow P(\ulcorner\psi(\dot{x})\urcorner))$.

If $\varphi(x)$ is a $\Pi_{n}$-formula such that $T \vdash \forall x . P_{T}^{h}(\ulcorner\varphi(\dot{x})\urcorner)$, then $T+C^{\circ} n_{P} \vdash$ $\forall x . \varphi(x)$.

Proof. As $\neg \varphi(x)$ is $\Sigma_{n}$, by $\mathbf{C 2} T \vdash \neg \varphi(x) \rightarrow P(\ulcorner\neg \varphi(\dot{x})\urcorner)$. Thus, $T \vdash$ $\neg P(\ulcorner\neg \varphi(\dot{x})\urcorner) \rightarrow \varphi(x)$. By $\mathbf{C 3}$, we have that $T+P\left(\left\ulcorner P_{T}^{h}(\ulcorner\varphi(\dot{x})\urcorner)\right\urcorner\right) \wedge$ $P\left(\left\ulcorner\neg P_{T}^{h}(\ulcorner\varphi(\dot{\dot{x}})\urcorner)\right\urcorner\right) \vdash P(\ulcorner\perp\urcorner)$, since $\neg \varphi:=\varphi \rightarrow \perp$. Hence, $T+\operatorname{Con}_{P} \vdash$ $P\left(\left\ulcorner P_{T}^{h}(\ulcorner\varphi(\dot{\dot{x}})\urcorner)\right\urcorner\right) \rightarrow \neg P\left(\left\ulcorner\neg P_{T}^{h}(\ulcorner\varphi(\dot{\dot{x}})\urcorner)\right\urcorner\right)$. Together with $\mathbf{C 1}$ we get that $T+\operatorname{Con}_{P} \vdash P\left(\left\ulcorner P_{T}^{h}(\ulcorner\varphi(\dot{x})\urcorner)\right\urcorner\right) \rightarrow \neg P(\ulcorner\neg \varphi(\dot{x})\urcorner)$, but, by what was previously concluded, one gets that $T+\operatorname{Con}_{P} \vdash P\left(\left\ulcorner P_{T}^{h}(\ulcorner\varphi(\dot{\dot{x}})\urcorner)\right\urcorner\right) \rightarrow \varphi(x)$. Suppose that $T \vdash \forall x . P_{T}^{h}(\ulcorner\varphi(\dot{x})\urcorner)$. As $h$ is primitive-recursive, we have that $P_{T}^{h}(x)$ is $\Sigma_{1}$. Ergo, by $\mathbf{C 2}, T \vdash P_{T}^{h}(\ulcorner\varphi(\dot{x})\urcorner) \rightarrow P\left(\left\ulcorner P_{T}^{h}(\ulcorner\varphi(\dot{\dot{x}})\urcorner)\right\urcorner\right)$. By assumption, it follows that $T \vdash \forall x . P\left(\left\ulcorner P_{T}^{h}(\ulcorner\varphi(\dot{\dot{x}})\urcorner)\right\urcorner\right)$; therefore, $T \vdash$ $\forall x . \varphi(x)$.

In the next result, we drop the assumption that $h$ is primitive-recursive, but we need to strengthen condition $\mathbf{C 1}$.

Theorem 5.3. Let $T$ be such that there is a provability predicate $P(x)$ satisfying:

C1 For all formulas $\varphi(x), T \vdash P_{T}^{h}(\ulcorner\varphi(\dot{x})\urcorner) \rightarrow P(\ulcorner\varphi(\dot{x})\urcorner)$;

C2 If $\varphi(x)$ is a $\Sigma_{n}$-formula, then $T \vdash \varphi(x) \rightarrow P(\ulcorner\varphi(\dot{x})\urcorner)$;

C3 For all formulas $\varphi(x)$ and $\psi(x), \quad T \vdash P(\ulcorner\varphi(\dot{x}) \rightarrow \psi(\dot{x})\urcorner) \rightarrow$ $(P(\ulcorner\varphi(\dot{x})\urcorner) \rightarrow P(\ulcorner\psi(\dot{x})\urcorner))$.

If $\varphi(x)$ is a $\Pi_{n}$-formula such that $T \vdash \forall x \cdot P_{T}^{h}(\ulcorner\varphi(\dot{x})\urcorner)$, then $T+C n_{P} \vdash$ $\forall x . \varphi(x)$.

Proof. As $\varphi(x)$ is $\Pi_{n}$, by $\mathbf{C 2}, T \vdash \exists x . \neg \varphi(x) \rightarrow \exists x . P(\ulcorner\neg \varphi(\dot{x})\urcorner)$. As $T \vdash$ $\forall x . P_{T}^{h}(\ulcorner\varphi(\dot{x})\urcorner)$, it follows, by $\mathbf{C 1}$, that $T \vdash \forall x . P(\ulcorner\varphi(\dot{x})\urcorner)$. This, together with the fact that $T+\exists x . \neg \varphi(x) \vdash \exists x . P(\ulcorner\neg \varphi(\dot{x})\urcorner)$, yields $T+\exists x . \neg \varphi(x) \vdash$ $\exists x . P(\ulcorner\neg \varphi(\dot{x})\urcorner) \wedge P(\ulcorner\varphi(\dot{x})\urcorner)$. As $\neg \varphi:=\varphi \rightarrow \perp$, it follows by $\mathbf{C 3}$ that $T+$ $\exists x . \neg \varphi(x) \vdash \exists x . P(\ulcorner\perp\urcorner)$, i.e., $T+\exists x . \neg \varphi(x) \vdash P(\ulcorner\perp\urcorner)$. Hence, $T+\operatorname{Con}_{P} \vdash$ $\forall x . \varphi(x)$.

Feferman, in [4], requires an intensionally correct arithmetization of provability to satisfy several conditions including $\mathbf{C 1}, \mathbf{C 2}$, and $\mathbf{C 3}$.

COROLLARY 5.2. Let $T$ be such that there is a provability predicate $P(x)$ satisfying:

C1 For all formulas $\varphi(x), T \vdash P_{T}^{h}(\ulcorner\varphi(\dot{x})\urcorner) \rightarrow P(\ulcorner\varphi(\dot{x})\urcorner)$; 
C2 If $\varphi(x)$ is a $\Sigma_{1}$-formula, then $T \vdash \varphi(x) \rightarrow P(\ulcorner\varphi(\dot{x})\urcorner)$;

C3 For all formulas $\varphi(x)$ and $\psi(x), \quad T \vdash P(\ulcorner\varphi(\dot{x}) \rightarrow \psi(\dot{x})\urcorner) \rightarrow$ $(P(\ulcorner\varphi(\dot{x})\urcorner) \rightarrow P(\ulcorner\psi(\dot{x})\urcorner)) ;$

C4 $T \vdash \operatorname{Con}_{P}$.

If $\varphi(x)$ is a $\Pi_{1}$-formula such that $T \vdash \forall x . P_{T}^{h}(\ulcorner\varphi(\dot{x})\urcorner)$, then $T \vdash \forall x . \varphi(x)$.

Proof. Follows immediately from the previous theorem.

By [4, 8], there is a provability predicate that satisfies $\mathbf{C 2}, \mathbf{C 3}$, and $\mathbf{C 4}$. Furthermore, if $P(x)$ is a provability predicate that satisfies $\mathbf{C} 2$ and $\mathbf{C 4}$, then $P^{\prime}(x):=P_{T}^{h}(x) \vee P(x)$ is a provability predicate that satisfies $\mathbf{C 1}, \mathbf{C 2}$, and C4. For this reason, we believe that any sufficiently strong theory $T$ satisfies all the previous conditions.

Using the theory $K_{T}$ we can go even further:

COROLLARY 5.3. Let $T$ be a theory in the conditions of the previous result. If $\varphi(x)$ is a $\Pi_{1}$-formula such that, for all $n \in \mathbb{N}, T \vdash_{\leq h} \varphi(\bar{n})$, then $K_{T} \vdash$ $\forall x . \varphi(x)$.

Proof. By the proof of Corollary 5.2, it can be concluded that $T \vdash$ $\forall x . P_{T}^{h}(\ulcorner\varphi(\dot{x})\urcorner) \rightarrow \forall x . \varphi(x)$. Thus, the result follows from Theorem 3.1. $\dashv$

A result similar to Theorem 5.3, for some $\Sigma$-formulas, holds in the presence of the stronger schema $\omega-\operatorname{Con}_{P}^{n}$ :

$$
P(\ulcorner\exists x . \varphi(x, \dot{y})\urcorner) \rightarrow \exists x . \neg P(\ulcorner\neg \varphi(\dot{x}, \dot{y})\urcorner), \quad \varphi(x) \text { is a } \Pi_{n} \text {-formula. }
$$

THEOREM 5.4. Let $T$ be such that there is a provability predicate $P(x)$ satisfying:

C1 For all formulas $\varphi(x), T \vdash P_{T}^{h}(\ulcorner\varphi(\dot{x})\urcorner) \rightarrow P(\ulcorner\varphi(\dot{x})\urcorner)$;

C2 For all $\Sigma_{n}$-formulas $\varphi(x, y), T \vdash \varphi(x, y) \rightarrow P(\ulcorner\varphi(\dot{x}, \dot{y})\urcorner)$.

Suppose that $\varphi(x)$ is a $\Pi_{n}$-formula. If $T \vdash \forall y . P_{T}^{h}(\ulcorner\exists x . \varphi(x, \dot{y})\urcorner)$, then $T+$ $\omega-\operatorname{Con}_{P}^{n} \vdash \forall y \cdot \exists x . \varphi(x, y)$.

Proof. Suppose that $T \vdash \forall y \cdot P_{T}^{h}(\ulcorner\exists x \cdot \varphi(x, \dot{y})\urcorner)$. By C1, we have $T \vdash$ $\forall y . P(\ulcorner\exists x . \varphi(x, \dot{y})\urcorner)$. Hence, $T+\omega-\operatorname{Con}_{P}^{n} \vdash \forall y . \exists x . \neg P(\ulcorner\neg \varphi(\dot{x}, \dot{y})\urcorner)$, i.e., $T+\omega-\operatorname{Con}_{P}^{n} \vdash \neg \exists y \cdot \forall x \cdot P(\ulcorner\neg \varphi(\dot{x}, \dot{y})\urcorner)$. Furthermore, by C2, we have $T+\exists y . \forall x . \neg \varphi(x, y) \vdash \exists y . \forall x . P(\ulcorner\neg \varphi(\dot{x}, \dot{y})\urcorner)$. Therefore, $T+\omega-\operatorname{Con}_{P}^{n} \vdash$ $\neg \exists y . \forall x . \neg \varphi(x, y)$, and so, $T+\omega-\operatorname{Con}_{P}^{n} \vdash \forall y \cdot \exists x . \varphi(x, y)$.

If $T \vdash \omega-\mathrm{Con}_{P}^{n}$, then everything is provable in $T$. We can yet get a stronger result, but, like before, we need a stronger schema. Let $\omega-\operatorname{Con}_{P}^{3, n}$ be the following schema:

$$
P(\ulcorner\exists y . \varphi(\dot{x}, y, \dot{z})\urcorner) \rightarrow \exists y . \neg P(\ulcorner\neg \varphi(\dot{x}, \dot{y}, \dot{z})\urcorner), \quad \varphi(x) \text { is a } \Pi_{n} \text {-formula. }
$$

THEOREM 5.5. Let $T$ be such that there is a provability predicate $P(x)$ satisfying:

C1 For all formulas $\varphi(x), T \vdash P_{T}^{h}(\ulcorner\varphi(\dot{x})\urcorner) \rightarrow P(\ulcorner\varphi(\dot{x})\urcorner)$; 
C2 For all $\Pi_{n}$-formulas $\varphi(x, y, z), \quad T \vdash P(\ulcorner\forall x . \exists y . \varphi(x, y, \dot{z})\urcorner) \rightarrow$ $\forall x . P(\ulcorner\exists y . \varphi(\dot{x}, y, \dot{z})\urcorner)$;

C3 For all $\Sigma_{n}$-formulas $\varphi(x, y, z), T \vdash \varphi(x, y, z) \rightarrow P(\ulcorner\varphi(\dot{x}, \dot{y}, \dot{z})\urcorner)$.

Suppose that $\varphi(x)$ is a $\Pi_{n}$-formula. If $T \vdash \forall z . P_{T}^{h}(\ulcorner\forall x . \exists y . \varphi(x, y, \dot{z})\urcorner)$, then $T+\omega-\operatorname{Con}_{P}^{3, n} \vdash \forall z . \forall x . \exists y \cdot \varphi(x, y, z)$.

Proof. $T+\forall z . P_{T}^{h}(\ulcorner\forall x . \exists y . \varphi(x, y, \dot{z})\urcorner) \vdash \forall z . P(\ulcorner\forall x . \exists y . \varphi(x, y, \dot{z})\urcorner), \quad$ by C1. From C2, $T+\forall z . P_{T}^{h}(\ulcorner\forall x . \exists y . \varphi(x, y, \dot{z})\urcorner) \vdash \forall z . \forall x . P(\ulcorner\exists y . \varphi(\dot{x}, y, \dot{z})\urcorner)$. This means that $T+\omega-\operatorname{Con}_{P}^{3, n} \vdash \forall z . \forall x . \exists y . \neg P(\ulcorner\neg \varphi(\dot{x}, \dot{y}, \dot{z})\urcorner)$, i.e., $T+\omega-\operatorname{Con}_{P}^{3, n} \vdash \neg \exists z \cdot \exists x . \forall y . P(\ulcorner\neg \varphi(\dot{x}, \dot{y}, \dot{z})\urcorner)$. As $\varphi(x, y, z)$ is $\Pi_{n}$, by C3, $\quad T+\exists z . \exists x . \forall y . \neg \varphi(x, y, z) \vdash \exists z . \exists x . \forall y . P(\ulcorner\neg \varphi(\dot{x}, \dot{y}, \dot{z})\urcorner)$. Altogether, $T+\omega-\operatorname{Con}_{P}^{3, n} \vdash \neg \exists z . \exists x . \forall y . \neg \varphi(x, y, z)$.

By Theorem 3.5, we have that the Local Reflection Principle (see [16, p. 845]) of $P_{T}^{h}(x)$ is provable in $T$, i.e., $T \vdash P_{T}^{h}(\ulcorner\varphi\urcorner) \rightarrow \varphi$. In fact, we have the following result.

THEOREM 5.6. Suppose that $h$ is primitive-recursive. Let $T$ be such that there is a provability predicate $P(x)$ satisfying:

C1 For all formulas $\varphi(x), T \vdash P_{T}^{h}(\ulcorner\varphi(\dot{x})\urcorner) \rightarrow P(\ulcorner\varphi(\dot{x})\urcorner)$;

C2 For all $\Sigma_{1}$-formulas $\varphi(x), T \vdash \varphi(x) \rightarrow P(\ulcorner\varphi(\dot{x})\urcorner)$;

C3 For all formulas $\varphi(x)$ and $\psi(x), \quad T \vdash P(\ulcorner\varphi(\dot{x}) \rightarrow \psi(\dot{x})\urcorner) \rightarrow$ $(P(\ulcorner\varphi(\dot{x})\urcorner) \rightarrow P(\ulcorner\psi(\dot{x})\urcorner)) ;$

C4 $T \vdash \operatorname{Con}_{P}$;

C5 For all formulas $\varphi(x), T \vdash \varphi(x) \Longrightarrow T \vdash P(\ulcorner\varphi(\dot{x})\urcorner)$.

Then, $T \vdash \forall x . P\left(\left\ulcorner P_{T}^{h}(\ulcorner\varphi(\dot{\dot{x}})\urcorner) \rightarrow \varphi(\dot{x})\right\urcorner\right)$.

Proof. As $h$ is primitive-recursive, we know that $P_{T}^{h}(x)$ and $\neg P_{T}^{h}(x)$ are $\Sigma_{1}$-formulas. By $\mathbf{C 2}, T \vdash P_{T}^{h}(\ulcorner\varphi(\dot{x})\urcorner) \rightarrow P\left(\left\ulcorner P_{T}^{h}(\ulcorner\varphi(\dot{x})\urcorner)\right\urcorner\right)$, so $T \vdash$ $\neg P\left(\left\ulcorner P_{T}^{h}(\ulcorner\varphi(\dot{\dot{x}})\urcorner)\right\urcorner\right) \rightarrow \neg P_{T}^{h}(\ulcorner\varphi(\dot{x})\urcorner)$. It holds that $T \vdash \neg P_{T}^{h}(\ulcorner\varphi(\dot{x})\urcorner) \rightarrow$ $P\left(\left\ulcorner\neg P_{T}^{h}(\ulcorner\varphi(\dot{\dot{x}})\urcorner)\right\urcorner\right)$. So, $T \vdash \neg P\left(\left\ulcorner P_{T}^{h}(\ulcorner\varphi(\dot{\dot{x}})\urcorner)\right\urcorner\right) \rightarrow P\left(\left\ulcorner\neg P_{T}^{h}(\ulcorner\varphi(\dot{\dot{x}})\urcorner)\right\urcorner\right)$, i.e., $T \vdash P\left(\left\ulcorner P_{T}^{h}(\ulcorner\varphi(\dot{\dot{x}})\urcorner)\right\urcorner\right) \vee P\left(\left\ulcorner\neg P_{T}^{h}(\ulcorner\varphi(\dot{\dot{x}})\urcorner)\right\urcorner\right)$.

From logic, $T \vdash \neg P_{T}^{h}(\ulcorner\varphi(\dot{x})\urcorner) \rightarrow\left(P_{T}^{h}(\ulcorner\varphi(\dot{x})\urcorner) \rightarrow \varphi(x)\right)$. Hence, by C5, $\quad T \vdash P\left(\left\ulcorner\neg P_{T}^{h}(\ulcorner\varphi(\dot{\dot{x}})\urcorner) \rightarrow\left(P_{T}^{h}(\ulcorner\varphi(\dot{\dot{x}})\urcorner) \rightarrow \varphi(\dot{x})\right)\right\urcorner\right) ; \quad$ thus, by $\mathbf{C 3}$, $T \vdash P\left(\left\ulcorner\neg P_{T}^{h}(\ulcorner\varphi(\dot{\dot{x}})\urcorner)\right\urcorner\right) \rightarrow P\left(\left\ulcorner P_{T}^{h}(\ulcorner\varphi(\dot{\dot{x}})\urcorner) \rightarrow \varphi(\dot{x})\right\urcorner\right)$.

From C1, $T+\neg P(\ulcorner\varphi(\dot{x})\urcorner) \vdash \neg P_{T}^{h}(\ulcorner\varphi(\dot{x})\urcorner)$. By C2, $T+\neg P(\ulcorner\varphi(\dot{x})\urcorner) \vdash$ $P\left(\left\ulcorner\neg P_{T}^{h}(\ulcorner\varphi(\dot{\dot{x}})\urcorner)\right\urcorner\right)$. Ergo we have $T+P\left(\left\ulcorner P_{T}^{h}(\ulcorner\varphi(\dot{\dot{x}})\urcorner)\right\urcorner\right)+\neg P(\ulcorner\varphi(\dot{x})\urcorner) \vdash$ $P\left(\left\ulcorner\neg P_{T}^{h}(\ulcorner\varphi(\dot{\dot{x}})\urcorner)\right\urcorner\right) \wedge P\left(\left\ulcorner P_{T}^{h}(\ulcorner\varphi(\dot{\dot{x}})\urcorner)\right\urcorner\right)$. From $\mathbf{C 3}$ and $\mathbf{C 4}$, it follows that $T+P\left(\left\ulcorner P_{T}^{h}(\ulcorner\varphi(\dot{\dot{x}})\urcorner)\right\urcorner\right)+\neg P(\ulcorner\varphi(\dot{x})\urcorner) \vdash \perp$, i.e., $T \vdash P\left(\left\ulcorner P_{T}^{h}(\ulcorner\varphi(\dot{\dot{x}})\urcorner)\right\urcorner\right) \rightarrow$ $P(\ulcorner\varphi(\dot{x})\urcorner)$. From logic, $T \vdash \varphi(x) \rightarrow\left(P_{T}^{h}(\ulcorner\varphi(\dot{x})\urcorner) \rightarrow \varphi(x)\right)$; thus, by 
C5 and C3, $T \vdash P(\ulcorner\varphi(\dot{x})\urcorner) \rightarrow P\left(\left\ulcorner P_{T}^{h}(\ulcorner\varphi(\dot{\dot{x}})\urcorner) \rightarrow \varphi(\dot{x})\right\urcorner\right)$. Hence, $T \vdash$ $P\left(\left\ulcorner P_{T}^{h}(\ulcorner\varphi(\dot{\dot{x}})\urcorner)\right\urcorner\right) \rightarrow P\left(\left\ulcorner P_{T}^{h}(\ulcorner\varphi(\dot{\dot{x}})\urcorner) \rightarrow \varphi(\dot{x})\right\urcorner\right)$.

So we have $T \vdash P\left(\left\ulcorner P_{T}^{h}(\ulcorner\varphi(\dot{\dot{x}})\urcorner)\right\urcorner\right) \vee P\left(\left\ulcorner\neg P_{T}^{h}(\ulcorner\varphi(\dot{\dot{x}})\urcorner)\right\urcorner\right)$ and also $T \vdash$ $P\left(\left\ulcorner\neg P_{T}^{h}(\ulcorner\varphi(\dot{\dot{x}})\urcorner)\right\urcorner\right) \rightarrow P\left(\left\ulcorner P_{T}^{h}(\ulcorner\varphi(\dot{\dot{x}})\urcorner) \rightarrow \varphi(\dot{x})\right\urcorner\right)$. From before, we have $T \vdash P\left(\left\ulcorner P_{T}^{h}(\ulcorner\varphi(\dot{\dot{x}})\urcorner)\right\urcorner\right) \rightarrow P\left(\left\ulcorner P_{T}^{h}(\ulcorner\varphi(\dot{\dot{x}})\urcorner) \rightarrow \varphi(\dot{x})\right\urcorner\right)$, and thus the result follows.

Inspired by the previous fact, one can consider the uniform reflection principle schema, $\operatorname{RFN}^{h}(T)$, for the provability notion $P_{T}^{h}(x)$ (see [16, $\mathrm{p}$. 845]):

$$
\forall x .\left(P_{T}^{h}(\ulcorner\varphi(\dot{x})\urcorner) \rightarrow \varphi(x)\right), \quad \varphi(x) \text { has only } x \text { free. }
$$

With $\Gamma$ being an arbitrary class of formulas (for example, $\Sigma_{n}, \Pi_{n}$, or even $\Delta_{n}$ ), we denote by $\operatorname{RFN}_{\Gamma}^{h}(T)$ the previous schema restricted to $\Gamma$-formulas and define $T_{\Gamma}^{h}:=K_{T}+\operatorname{RFN}_{\Gamma}^{h}(T)$. There is a deep relation between $\omega$ consistency and reflection principles [16, p. 853]: Restrictions to П-formulas of the uniform reflexion principle for the standard provability predicate are equivalent to restrictions of the schema $\omega-\operatorname{Con}_{\mathrm{Pr}}^{n}$ from above to $\Sigma$-formulas. Note that we are adding $\omega$-consistency and not $\omega$-completeness; hence Kreisel's conjecture - which follows immediately from $\omega$-completeness - is not being trivialised.

Now we presented another adapted version of Kreisel's conjecture.

THEOREM 5.7. Let $h$ be a total recursive function and $\varphi(x)$ be a $\Gamma$-formula such that, for all $n \in \mathbb{N}, T \vdash_{\leq h} \varphi(\bar{n})$. Then, $T_{\Gamma}^{h} \vdash \forall x . \varphi(x)$.

Proof. Let $h$ be a total recursive function and $\varphi(x)$ be a $\Gamma$-formula such that, for all $n \in \mathbb{N}$, PA $\vdash_{\leq h} \varphi(\bar{n})$. By Theorem 3.1, we have that $K_{T} \vdash$ $\forall x . P_{T}^{h}(\ulcorner\varphi(\dot{x})\urcorner)$. Thus, by $\operatorname{RFN}_{\Gamma}^{h}(T)$, it follows that $T_{\Gamma}^{h} \vdash \forall x . \varphi(x)$.

Note that there are no particular reasons to believe that the theory $K_{T}$ is effectively axiomatisable. This is something worth studying.

Furthermore, one could consider a modal logic with modalities (interpreted by $\left.\operatorname{Pr}_{\mathrm{PA}}(\cdot)\right)$ and $\square_{\leq h}$ (with $P_{T}^{h}(\cdot)$ as an intended interpretation) and, at least, the usual axioms of $\square$ and the properties of $P_{T}^{h}(\cdot)$. For example, as modal versions of Theorems 3.3, 3.5, and 3.6, one could add the following axioms:

Ax.1 $\left(\square \square_{\leq h} A\right) \vee\left(\square \neg \square_{\leq h} A\right)$;

Ax.2 $\square_{\leq h} \bar{A} \rightarrow A$;

Ax.3 $\neg \square \neg \square_{\leq h} A \rightarrow \square_{\leq h} A$.

§6. On $\vdash_{k \text { steps }}$ and $\vdash_{\leq h}$. From [3, p. 8], we know the following fact:

THeOREM 6.1. If $T$ is a finitely axiomatised theory, then there is a total recursive function $f(k, \# \varphi)$ such that

$$
T \vdash_{k \text { steps }} \varphi \Longrightarrow T \vdash_{f(k, \# \varphi) \text { symbols }} \varphi .
$$

With this theorem, one can establish a relation between $\vdash_{k \text { steps }}$ and $\vdash_{\leq h}$. 
THEOREM 6.2. Given $k$, if $T$ is a finitely axiomatised theory, then the function

$$
g_{k}(\# \varphi):= \begin{cases}1, & T \vdash_{k \text { steps }} \varphi \\ 0, & \text { otherwise }\end{cases}
$$

is recursive.

Proof. Let $k$ be fixed. We will intuitively describe the algorithm that computes the function $g_{k}$. Consider the input \# $\varphi$. Compute, by Theorem 6.2, $f(k, \# \varphi)$. If $\varphi$ is provable with at most $k$ steps, then it must be provable using at most $f(k, \# \varphi)$ symbols. In such a hypothetical proof, clearly there are, at most, $f(k, \# \varphi)$ different variables. Furthermore, the variables, besides the ones that occur in $\varphi$, can be arbitrarily chosen, i.e., if one performs a change of variables in the proof without changing the variables occurring in $\varphi$, one maintains the soundness of the proof and the number of steps in it. This means that it is enough to consider a finite set of variables consisting of: the variables in $\varphi$ and $f(k, \# \varphi)$ other variables. Then, the algorithm considers all possible finite strings constructed using the finite set consisting of: the logical connectives, quantifiers, parenthesis, a blank symbol (to separate the steps in a proof), and the variables of the finite set that was mentioned. By vanishing over all the (finite) possible strings, the algorithm tests if any of them is a proof of $\varphi$ with at most $k$ steps. If there is any, it outputs 1; otherwise it ought to output 0 . Thus, the algorithm outputs 1 exactly when $\varphi$ is provable with at most $k$ steps.

THEOREM 6.3. Given $k$, if $T$ is a finitely axiomatised theory, then there is a total recursive function $h_{k}$ such that

$$
T \vdash_{k \text { steps }} \varphi \Longrightarrow T \vdash_{\leq h_{k}} \varphi .
$$

Proof. Let $g_{k}$ be as in Theorem 6.2. It is immediate that the function

$$
h_{k}(n):= \begin{cases}m, & \text { if } g_{k}(n)=1 \text { and } m \text { is the smallest code of a proof of the } \\ & \text { formula coded by } n \text { with at most } k \text { steps } \\ 0, & \text { otherwise }\end{cases}
$$

is total recursive. We show that $T \vdash_{k \text { steps }} \varphi \Longrightarrow T \vdash_{\leq_{k}} \varphi$. If $T \vdash_{k \text { steps }}$, then $g_{k}(n)=1$ and so $h_{k}(\# \varphi)$ is the code of a proof of $\varphi$ with at most $k$ steps; by definition, $T \vdash_{\leq h_{k}} \varphi$.

There are two immediate consequences of the previous result.

Corollary 6.1. Suppose that $T$ is a finitely axiomatised theory satisfying the conditions of Corollary 5.3 for the function $h_{k}$ and that $\varphi(x)$ is a $\Pi_{1-}$ formula. If for all $n \in \mathbb{N}, T \vdash_{k \text { steps }} \varphi(\bar{n})$, then $K_{T} \vdash \forall x . \varphi(x)$.

PROof. Follows from the previous theorem and from Corollary 5.3. $\dashv$

Corollary 6.2. Suppose that $T$ is a finitely axiomatised theory and that $\varphi(x)$ be a $\Gamma$-formula. If, for all $n \in \mathbb{N}, T \vdash_{k \text { steps }} \varphi(\bar{n})$, then $T_{\Gamma}^{h_{k}} \vdash \forall x . \varphi(x)$. 
Proof. Follows from Theorems 5.7 and 6.3.

We finish with an open problem.

PROBLEM. Is there a total recursive function $h$ such that, for all formulas $\varphi, \mathrm{PA} \vdash_{k \text { steps }} \varphi \Longrightarrow \mathrm{PA} \vdash_{\leq h} \varphi$ ?

\$7. Conclusion. Kreisel's conjecture is the fundamental problem of $k$ steps-provability. As mentioned in the introduction, there are some solutions under specific conditions. Usually they rely on properties of the considered formulas or properties of the theory. In this paper, we presented a novel approach to the conjecture, where we abstracted from the concrete formalization.

We introduced a notion of provability $\vdash_{\leq h}$ expressing that $T \vdash_{\leq h} \varphi$ holds if there is a proof of $\varphi$ in $T$ whose code is at most $h(\# \varphi)$. This is clearly an intensional notion. We studied the representation of $\vdash_{<h}$ inside the theory $T$ using the formula $P_{T}^{h}(x)$ and several of its properties. Montagna's conjecture ("Does PA $\vdash_{k \text { steps }} \operatorname{Pr}_{\mathrm{PA}}(\ulcorner\varphi\urcorner) \rightarrow \varphi$ imply PA $\vdash_{k \text { steps }} \varphi$ ?") was analysed for the notion $\vdash_{<h}$.

We also considered variants of Kreisel's conjecture for provability predicates with different derivability conditions. From the results, we like to highlight Theorem 5.4 that, using a form of $\omega$-consistency $\left(\omega-\operatorname{Con}_{P}^{n}\right)$ and under certain derivability conditions, allows to conclude $T+\omega-\operatorname{Con}_{P}^{n} \vdash$ $\forall y . \exists x . \varphi(x, y)$ from $T \vdash \forall y . P_{T}^{h}(\ulcorner\exists x . \varphi(x, \dot{y})\urcorner)$.

The paper finishes with connections between $\vdash_{k \text { steps }}$ and $\vdash_{\leq h}$, in particular, two forms of Kreisel's conjecture for $\vdash_{\leq h}$ (Corollaries 6.1 and 6.2).

Acknowledgments. This work was funded by the following FCT-projects: Centro de Matemática e Aplicações (UIDB/00297/2020) and Bolsa de Doutoramento (SFRH/BD/143756/2019). The second author was also supported by the Udo Keller Foundation.

\section{REFERENCES}

[1] M. BaAz and P. Pudlák, Kreisel's conjecture for L $\exists 1$, Arithmetic, Proof Theory and Computational Complexity (P. Clote and J. Krajićek, editors), Oxford University Press, Oxford, 1993, pp. 29-59.

[2] S. CAVAGNETTO, The lengths of proofs: Kreisel's conjecture and Gödel's speed-up theorem. Journal of Mathematical Sciences, vol. 158 (2009), no. 5, pp. 689-707.

[3] P. Clote and J. Krajićek, Open problems, Arithmetic, Proof Theory and Computational Complexity (P. Clote and J. Krajićek, editors), Oxford University Press, Oxford, 1993, pp. 1-19.

[4] S. Feferman, Arithmetization of metamathematics in a general setting. Fundamenta Mathematicae, vol. 49 (1960), no. 1, pp. 35-92.

[5] H. Friedman, One hundred and two problems in mathematical logic. The Journal of Symbolic Logic, vol. 40 (1975), no. 2, pp. 113-129.

[6] P. Hrubeš, Theories very close to PA where Kreisel's conjecture is false. The Journal of Symbolic Logic, vol. 72 (2007), no. 1, pp. 123-137.

[7] T. KURAHASHI, Arithmetical soundness and completeness for $\Sigma_{2}$ numerations. Studia Logica, vol. 106 (2018), no. 6, pp. 1181-1196. 
[8] - A note on derivability conditions. The Journal of Symbolic Logic, vol. 85 (2020), no. 3, pp. 1224-1253.

[9] P. LindströM, Aspects of Incompleteness, Lecture Notes in Logic 10, Cambridge University Press, Cambridge, 2017.

[10] S. Liv, An enumeration of the primitive recursive functions without repetition. Tohoku Mathematical Journal, Second Series, vol. 12 (1960), no. 3, pp. 400-402.

[11] T. Miyatake, On the length of proofs in a formal systems. Tsukuba Journal of Mathematics, vol. 4 (1980), no. 1, pp. 115-125.

[12] R. PARIKH, Some results on the length of proofs. Transactions of the American Mathematical Society, vol. 177 (1973), pp. 29-36.

[13] M. RathJen and W. Sieg, Proof theory, The Stanford Encyclopedia of Philosophy (E. N. Zalta, editor), Metaphysics Research Lab, Stanford University, Stanford, 2018, Appendix F. Provably Recursive Functions.

[14] R. Ritchie and P. Young, Strong representability of partial functions in arithmetic theories. Information Sciences, vol. 1 (1969), no. 2, pp. 189-204.

[15] P. SмITH, An Introduction to Gödel's Theorems, second ed., Cambridge Introductions to Philosophy, Cambridge University Press, Cambridge, 2013.

[16] C. SMORYNSKI, The incompleteness theorems, Handbook of Mathematical Logic, eighth ed., North-Holland, Amsterdam, 1993, pp. 821-865.

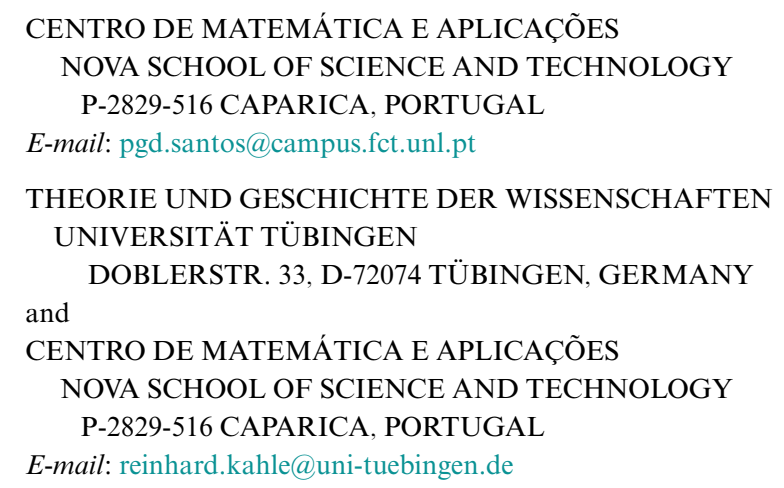

